\title{
ERRATUM
}

Tatsuya Ohkawara $\cdot$ Hiroshi Takeda

Kencho Miyashita - Morie Nishiwaki

Toshinori Nakayama • Masaru Taniguchi

Takashi Yoshiki · Junji Tanaka - Masahiro Imamura

Toshiro Sugiyama • Masahiro Asaka · Jun Nishihira

\section{Regulation of Toll-like receptor 4 expression in mouse colon by macrophage migration inhibitory factor}

Published online: 8 December 2005

(C) Springer-Verlag 2005

Due to an unfortunate error the name of one of the authors was misspelled. The author's name should read: Junji Tanaka.

The online version of the original article can be found at http:/ dx.doi.org/10.1007/s00418-005-0092-y

T. Ohkawara $(\bowtie) \cdot H$. Takeda $\cdot$ K. Miyashita $\cdot$ M. Asaka Department of Gastroenterology and Hematology,

Hokkaido University Graduate School of Medicine, Kita-15, Nishi-7, Kita-ku, 060-8638 Sapporo, Japan

E-mail: tokawara@med.hokudai.ac.jp

Tel.: + 81-11-7161161

Fax: $+81-11-7067867$

M. Nishiwaki $\cdot$ T. Yoshiki $\cdot$ J. Nishihira

Department of Research and Development,

GeneticLab, 060-8638 Sapporo, Japan

T. Nakayama

Department of Molecular Immunology, Graduate School of

Medicine, Chiba University, 263-8522 Chiba, Japan

M. Taniguchi

Department of Medical Immunology,

Graduate School of Medicine, Chiba University,

263-8522 Chiba, Japan

J. Tanaka $\cdot$ M. Imamura

Department of Hematology and Oncology, Hokkaido University Graduate School of Medicine, 067-8638 Sapporo, Japan

T. Sugiyama

Third Department of Internal Medicine, Toyama Medical and

Pharmaceutical University, 930-0194 Toyama, Japan 